\title{
Teaching Microprocessors Design Using FPGAs
}

\author{
Joaquín Olivares, José Manuel Palomares, José Manuel Soto, Juan Carlos Gámez \\ Dept. of Computer Architecture, Electronics, and Electronics Technology \\ University of Córdoba \\ Córdoba, Spain \\ olivares@uco.es
}

\begin{abstract}
Microprocessors is a typical subject within the Computer Architecture field of scope. It is quite common to use simulators in practical sessions, due to the complexity of its contents. In this paper a new methodology based on practical sessions with real devices and chips is proposed. Simple designs of microprocessors are exposed to the students at the beginning, rising the complexity gradually toward a final design with a multiprocessor integrated in a single FPGA chip. Finally, assessment results are shown.
\end{abstract}

Keywords: Learning Experiences; Laboratory Experiences; Teaching Engineering; Computer Architecture

\section{INTRODUCTION}

Traditional laboratory practical sessions to teach microprocessors are based on simulators, in this paper an experience implementing real microprocessors is shown. The main purpose is to encourage the student interest and to improve the assessment. This proposal is useful for many subjects within several engineering degrees. This activity was carried out in particular in the Microprocessors subject, in the degree in Computer Sciences at the University of Córdoba.

In most cases, computer architecture has been taught with software simulators [1], [2]. These simulators are useful to show: internal values in registers, memory accesses, cache fails, etc. However, the structure of the microprocessor is not visible, and students are not aware learning a real processor. Recently [3], [4], digital design is being teaching using real Programmable Logic Devices (PLD), showing it attractiveness for the students. Also recent works shown how the learning throw projects and games based on FPGAs are very useful [5].

In this work, a methodology for easy design and real implementation of microprocessors is proposed, in order to provide students with a user-friendly tool. Simple designs of microprocessors are exposed to the students at the beginning, rising the complexity gradually toward a final design with two processors integrated in an FPGA; each of which has an independent memory system, and are intercommunicated with a unidirectional serial channel. Furthermore, an introduction to the architecture of a T1 SUN OpenSparc system with 8 processors, 4 thread/processor plus one MicroBlaze is introduced at the end of the semester while students are working on their projects, this final seminar is useful because students are encouraged when see that a high performance parallel architecture is suitable of being implemented on a single FPGA Virtex5 [6].
In this paper the methodology to design and implement a microprocessor or multiprocesors is presented. To illustrate it with high detail and in a useful way, how to design the most complex practical session is shown. In section I, other methodologies based on simulators are referenced. The software platform used to implement real processors is presented in section II. Features of MicroBlaze processor are introduced in section III. The course practical content is described in section IV. To illustrate how a processor is designed and implemented, section $\mathrm{V}$ presents a brief guide of how to build a biprocessor, this is the most complex practical session. Finally, conclusions are presented in section VI.

\section{SOFTWARE TOOL}

The Xilinx Platform Studio (XPS) is used to design MicroBlaze processors. XPS is a graphical IDE for developing and debugging hardware and software. XPS simplifies the procedure to the users, allowing them to select, interconnect, and configure components of the final system. Dealing with this activity, the student learns to add processors and peripherals, to connect them through buses, to determine the processor memory extension and allocation, to define and connect internal and external ports, and to customize the configuration parameters of the components. Once the hardware platform is built, the students learn many concepts about the software layer, such as: assigning drivers to peripherals, including libraries, selecting the operative system (OS), defining processor and drivers parameters, assigning interruption drivers, establishing OS and libraries parameters.

Students have deeper documentation available in [7], [8], and other useful resources recommended are [9], and, [10].

An embedded system performed with XPS can be summarized as a conjunction of a Hardware Platform (HWP) and a Software Platform (SWP), each defined separately.

\section{A. The Hardware Platform}

The HWP is described in the Microprocessor Hardware Specification (MHS) file; it contains the description of the system architecture, the memory map and the configuration parameters. HWP can be defined as one or more processors connected to one or more peripherals through one or more buses. The definition of the activity follows this sequence:

- To add processors and peripherals.

- To connect them through buses. 


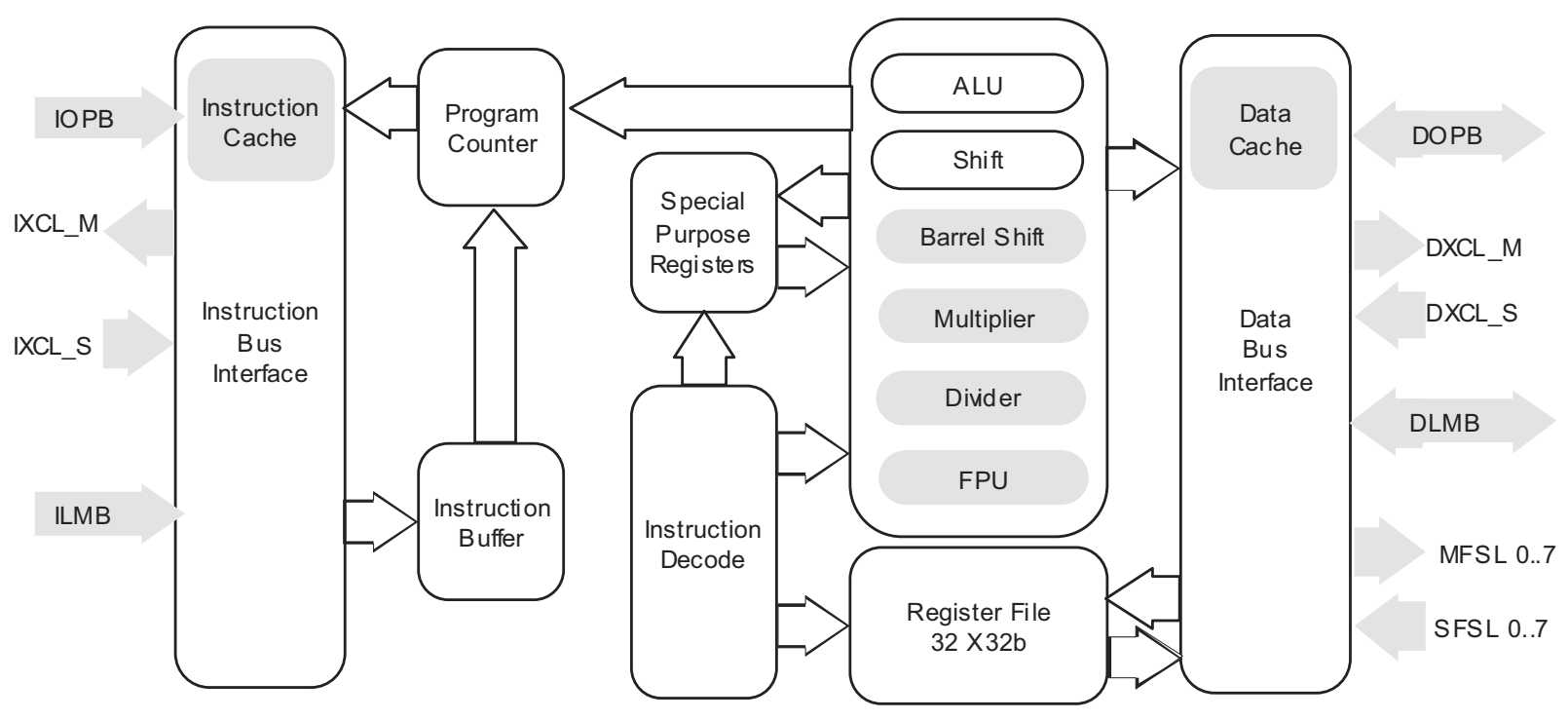

Legend Optional feature. This figure is inspired on MicroBlaze Processor Reference Guide. Xilinx. 2005

Figure 1. MicroBlaze architecture. In gray, reconfigurable components

- To determine the processor memory allocation.

- To define and connect internal and external ports.

- To customize the configuration parameters of the components.

\section{B. The Software Platform}

The SWP is described in the Microprocessor Software Specification (MSS) file; it contains the description of drivers, component libraries, configuration parameters, standard input/output devices, interruption routines and other software features. The sequence of activities needed to define the SWP is the following:

- To assign drivers to peripherals.

- To assign interruption drivers.

- To establish OS and libraries' parameters.

\section{The MicroBlaze Processor}

MicroBlaze is a 32-bit specific purpose processor developed by Xilinx in VHDL. It can be parameterized using XPS to obtain an $\grave{a}$-la-carte processor. It is a RISC processor, structured as a Harvard architecture with separated data and instruction interfaces.

MicroBlaze components are divided into two main groups depending on their configurability as shown in Fig.1.

Some fixed feature components are:

- 32 general purpose registers sized 32-bit each.

- Instructions with 32 bits word-sized, with 3 operands and 2 addressing modes.

- 32 bits address bus.
- 3-stage Pipeline.

Some of the most important configurable options are:

- An interface with OPB (On-chip Peripheral Bus) data bus.

- An interface with OPB instruction bus.

- An interface with LMB (Local Memory Bus) data bus.

- An interface with LMB instruction bus.

- Instruction cache.

- $\quad$ To include EDK libraries.

- To select the operative system (OS).

- To define processor and drivers' parameters.

- Data cache.

- 8 Fast Simplex Link (FSL bus) Interfaces.

- CacheLink bus support.

- Hardware exception support.

- $\quad$ Floating Point Unit (FPU).

\section{Practical Designs}

Practical sessions introduce gradual learning, allowing the fast design based on previous sessions. Essential problems in hardware programming will be raised:

- Hyperterminal serial communication.

- Using IO ports.

- Memory controller.

- Interruption routines and priority. 


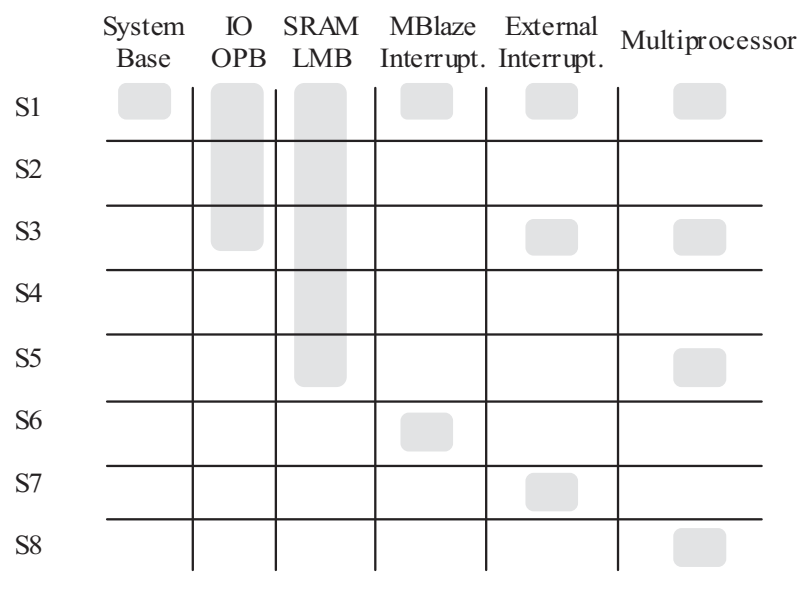

Figure 2. Sessions and contents.

- Message passing in multiprocessors communication.

The practical content of the subject is composed of 8 projects. In the first session, students make a basic system which will be used in following sessions as the base core system. Second and third sessions are used to introduce the input/output flow and the communication with external peripheral through the On-chip Peripheral Bus, for general purpose. SRAM external memory is added to the system at fourth session. Next session is dedicated to the External Memory Controller and how to split the bus. MicroBlaze interruptions are added in the sixth session, and external interruptions using the interruption controller are included in the seventh session. Finally, students build a biprocessor, using the Fast Simple Link channel at session eight. In fig. 2 the relation between practices is shown. For instance, $5^{\text {th }}$ session is based on all previous sessions, $7^{\text {th }}$ session is based on $3^{\text {rd }}$ and $1^{\text {st }}$ session.

\section{BIPROCESSOR SYSTEM DESIGN}

The last and most complex practical session is the design and implementation of a biprocessor. A computational system composed of two MicroBlazes will be designed. Both MicroBlazes will be interconnected using message-passing protocol. Each MicroBlaze has its own non-shared memory for instructions and data.

In the Fig. 3 a diagram with the structure of the design is shown. In it, the buses and components used have been detailed. It also includes how they are interconnected

At first, following the logical sequence exposed previously, a HWP will be created. This HWP will include the configuration of the components and buses, their interconnection, the memory map, ports and other parameters.

In the following subsection, the steps needed to configure the system will be described. Trivial stages, such as the creation of the project, will not be included in this paper. The parameters shown in this section depends on the FPGA chip, in this case the Spartan 3 board [11].

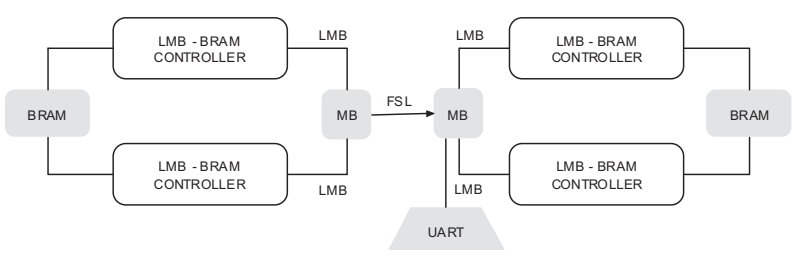

Figure 3. Biprocessor system diagram.

\section{A. Hardware Platform Specifications}

This stage is described in the MHS file. Following, the components specified in the structure of the system are enumerated:

- Two MicroBlaze processors.

- Two on-chip RAM memory blocks (BRAM), one for each processor.

- $\quad$ One UART.

- One OPB bus, to connect the UART with the slave processor.

- Two LMB buses to communicate each processor with their respective data memory controller; and another two LMB buses to interconnect the processors with their instruction memory controller.

- One FSL channel to intercommunicate each processor with the other.

After that, the interconnection of buses and components is defined. The connection of the memory ports are also set at this point. The student has to specify in the connection matrix which components are linked to which buses and with which kind of connection.

In the exposed case, four LMB buses are needed to access local memory, two for each MicroBlaze, because each processor has its own memory subsystem.

Also, one FSL channel which connects both processors.

Each BRAM has been designed with 4 different ports. Each MicroBlaze reaches its memory block through two different interfaces (instructions and data).

After that, it is necessary to map the components inside the configuration memory of the processors. XPS provides a functionality which is able to compute automatically a valid configuration memory map for a monoprocessor system structure. However, as the system proposed is a biprocessor one, this functionality cannot be used.

Each MicroBlaze looks for the first instruction in its program at memory address $0 \mathrm{x} 0$.

The next step is to define the internal and external ports. Most of the internal ones are configured by XPS with default settings. It is also necessary to define and to connect some of the internal ports to make the system works: those ports related to the reset and clock signals must be forwarded to all of the subsystems and components. Four external ports are mandatory: clock, reset, UART in and UART out. With these 
ports, the student sends commands and synchronization information to the system. Finally, the components are configured. The parameters for each component and their meaning are described thoroughly in the documentation included in the XPS platform.

Particularly, MicroBlaze includes a parameter which selects the amount of FSL interfaces used. Thus, both processors have to set this configuration value to one to allow the communication between them. The configuration of this parameter is done by changing C_FSL_LINKS. This parameter has to be set to a numerical value, representing the amount of FSL interfaces to be included in the core.

Another interesting configuration to be mentioned is the UART operational configuration. The student has to determine the operational frequency, the application of the parity bit checking, working bauds, etc. A valid set of parameters for the UART and MicroBlaze are the following:

\section{1) UART parameters}

a) $\quad$ C_CLK_FREQ $=50 \_000 \_000$. Set the frequency of the OPB bus, connected to the UART. It has to coincide with the operational system speed.

b) C_BAUDRATE $=19200$. Set the bauds for the UART. The terminal used to receive characters has to be configured at the same baud rate.

c) $C \backslash$ USE $\backslash$ PARITY $=0$. Set whether the UART should work with parity bit or not.

2) MicroBlaze parameters.

a) C_FSL_LINKS $=1$. In order to communicate between the two processors, at least, one FSL channel has to be defined.

After the HWP is defined, the netlist files and the support files can be generated.

\section{B. Constraints File}

The constraints file specifies how external ports from the designed system correspond with the Spartan-3 Board [ ] pins:

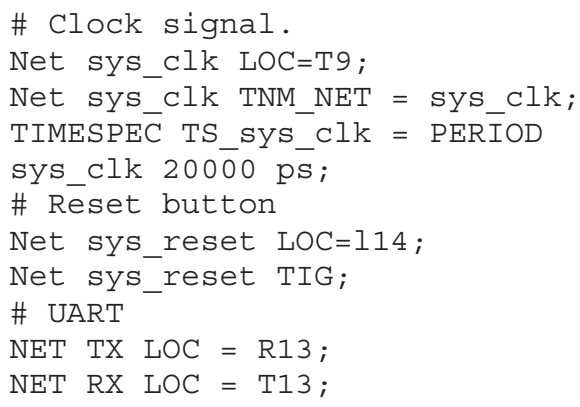

Once the constraint file and the HWP are ready, XPS has enough information to create the internal bitstream file.

\section{Software Platform Specification}

The SWP corresponds with the Microprocessor Software Specification (MSS). The first step is to select the drivers for
MBlaze 1

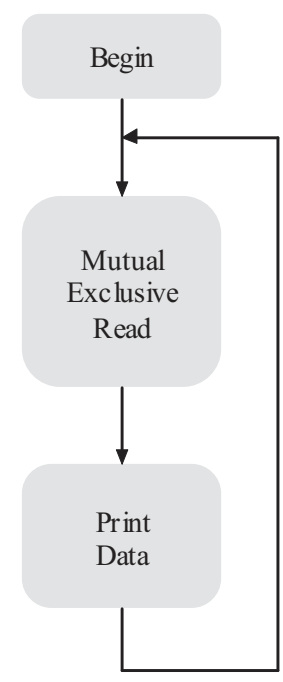

MBlaze 2

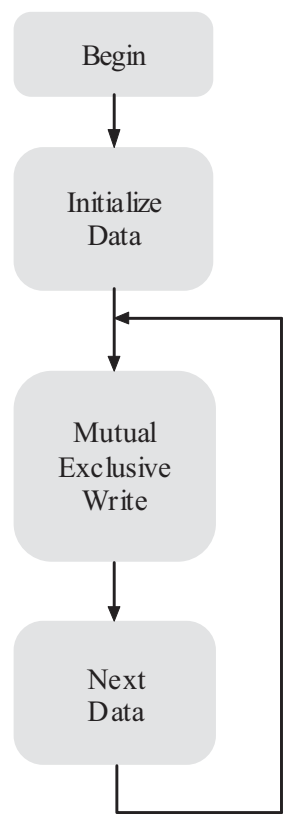

Figure 4. Software application flowchart.

the components, the SWP libraries and the OS for each processor. In this project, the standalone OS is selected, no libraries are used and the drivers for the components are established by default.

In the following step, the configurable parameters from processors and drivers must be edited; also the interruption routines must be assigned if needed; for instance, in the system under explanation, the interruption routines are not used. In this case, all fields are configured by default except for the processors, which must be configured to work at $100 \mathrm{MHz}$.

In the final step, the OS libraries configuration parameters are edited and configured. The standalone OS has a minimum size software layer with some primitives for input/output, control, memory allocation, etc.

In the proposed system, the student must specify the UART as the input/output standard peripheral. This is established by assigning the UART to the OPB master MicroBlaze.

Once SWP is configured, it is possible to program the processors software code. However, it is necessary to generate previously the libraries and the Board Support Package (BSP).

\section{Software Application}

To carry out the goals of this work it is necessary to create two SW projects, one for each MicroBlaze. With these, each Microblaze will execute its own program.

Some primitives are used to allow a fluid message passing between both processors through the FSL channel.

The writer processor generates the data that will be sent to the reader processor through the FSL channel using mutual exclusion primitives for message passing. The OPB bus master sends the data received by the reader processor to the UART. 


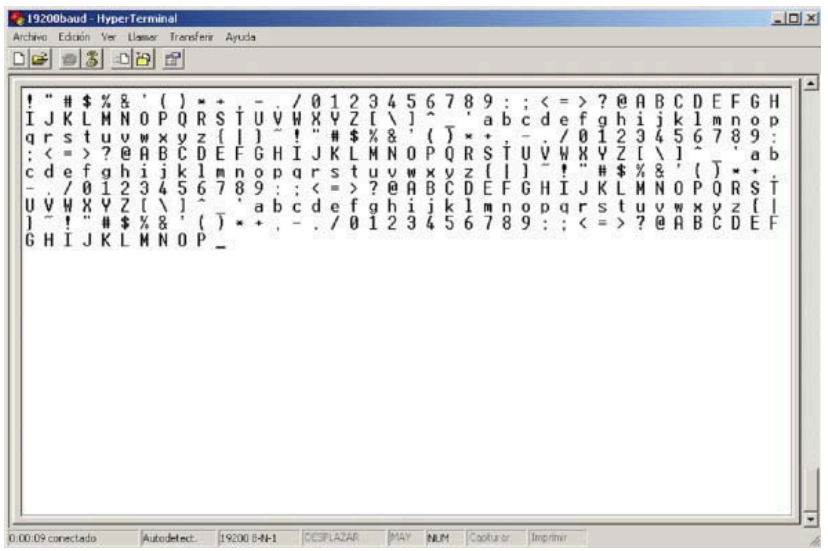

Figure 5. Hyperterminal capture while the both processors are

After the compilation of the software application, the FPGA configuration file is generated.

Finally, the configuration file is loaded into the FPGA. After all these steps, the students execute the global system and they verify that both processors work properly: The reader processor reads data generated from the writer processor, and the writer processor generates successive data. This process is shown in Fig. 4.

Following the code based on $\mathrm{C}$ language for both MicroBlaze processors is shown. Some Xilinx libraries are required to optimize the code and to use some resources:

\section{MicroBlaze 1 Code. Reading data from MicroBlaze 2 and showing them in the UART}

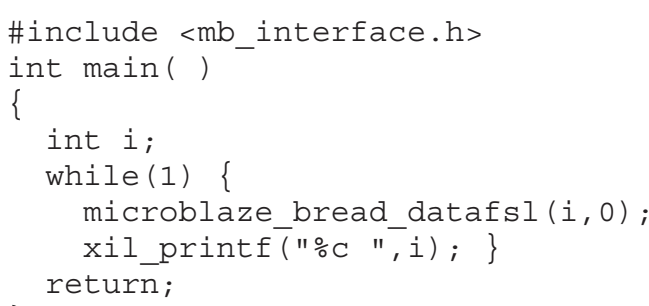

MicroBlaze 2 Code. Generating consecutive ascii characters and sending them to MicroBlaze 1 through FSL

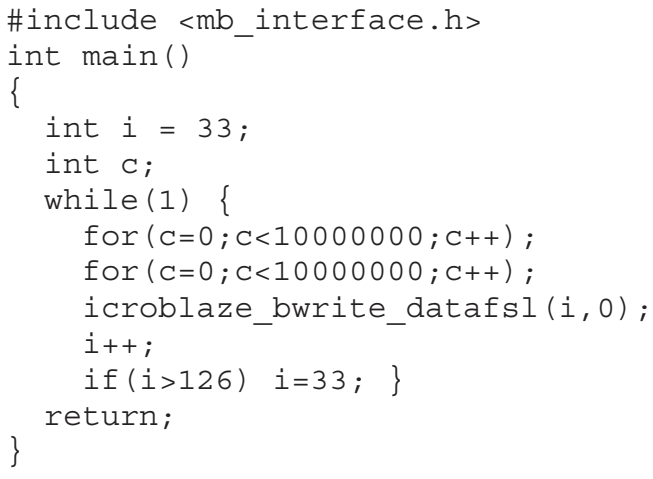

Hyperterminal screen was captured and is shown in Fig. 5 to show the results when the both processors are running.

\section{CONCLUSIONS}

XPS can be used as an excellent tool for the students to design and build complex architectures avoiding implementation details. Otherwise, they would spend a lot of time until they master concepts and techniques to develop those systems. Students understand the functionality and the structure of the different components, in order to interconnect all of them to build either a monoprocessor or a biprocessor.

A notable improvement of the qualifications compared to the average of the previous five years was obtained. In particular, in 2008 an improvement of $21 \%$ was obtained in front of the mean of the assessment for $2005-07$ period. In 2009 the improvement in the assessment was $22 \%$.

As a result of a survey, students are more motivated using real devices than using just simulators.

Furthermore, the configuration of each of the component parameters contributes to a better understanding of the developed architecture. And they are able to test how different values for those parameters influence the performance of the whole system. Finally, a guide on how to implement several processors systems on a single FPGA chip has been provided.

\section{ACKNOWLEDGMENT}

Authors wish to remark the great task carried out by the Xilinx University Program, XUP; and the Sun Microsystems OpenSPARC University Program, which have donated software and materials which have been very useful to partially finance this work.

\section{REFERENCES}

[1] R. D. Williams, R. H. Klenke, and J.H. Aylor. "Teaching Computer Design Using Virtual Prototyping," IEEE Trans. on Education, vol. 46, no. 2, 296-301, 2003.

[2] Simuproc. http://simuproc.softonic.com/ . Last accessed on $1^{\text {st }}$ September 2009.

[3] T. Weng, Y. Zhu, and C.-K. Cheng. "Digital Design and Programmable Logic Boards: Do Students Actually Learn More?," in Proc. ASEE/IEEE Frontiers in Education Conference, 2008.

[4] Y. Zhu, T. Weng, and C.-K. Cheng. "Enhancing Learning Effectiveness in Design Courses Through the Use of Programmable Logic Boards," IEEE Trans. on Education, vol. 52, no. 1, 151-156, 2009.

[5] V. Sklyarov, and I. Skliarova. "Teaching Reconfigurable Systems: Methods, Tools, Tutorials, and Projects," IEEE Trans. on Education, vol. 48, no. 2, 290-300, 2005.

[6] OpenSPARC http://www.opensparc.net/edu/university-program.html. Last accessed on $8^{\text {th }}$ November 2009.

[7] -. "Platform Studio User Guide," Application notes, Xilinx, 2005.

[8] -. "Microblaze Processor Reference Guide," Application notes, Xilinx, 2005.

[9] -. "Embedded System Tools Reference Manual," Application notes, Xilinx, 2008

[10] -. "OS and Libraries Document Collection," Xilinx, Application notes, September 2007.

[11] Spartan-3 Board. http://www.digilentinc.com/ . Last accessed on $30^{\text {th }}$ October 2009. 


\section{Authors Index}

\begin{tabular}{|c|c|}
\hline Abbas, Ali & 737 \\
\hline Abdelaoui, Nacer & 1937 \\
\hline Abu aisheh, Akram & 1425 \\
\hline Aedo, Ignacio & 1931 \\
\hline Agea, Álvaro & 1247 \\
\hline Agila Palacios, Martha V. & 217 \\
\hline Aguado, Luis Angel & 365 \\
\hline Aguiar, Javier M. & 77 \\
\hline Ahmed, Ibrahimi & 1801 \\
\hline Aledo, Angel & 365 \\
\hline Alexander, Brad & 997 \\
\hline Alimi, Adel & 401 \\
\hline Allert, Heidrun & 1597 \\
\hline Almeida Martínez, F.J. & 1019 \\
\hline Alonso Atienza, Felipe & 783,1263 \\
\hline Alonso Fernández, F. & 1181 \\
\hline Alorda, Bartomeu & 653 \\
\hline Al Smadi, Mohammad & 493 \\
\hline Álvarez Melcón, A. & 1195 \\
\hline Álvarez, Miguel Angel & 1493 \\
\hline Alvarez, Teresa & 1541 \\
\hline Alvarez Vellisco, A. & 237 \\
\hline Alves, Gustavo R. & 1359 \\
\hline Al Zoubi, Abdullah Y. & 1425 \\
\hline Amante García, Beatriz & 1335,1835 \\
\hline Anaya, Antonio R. & 797 \\
\hline Andrés Gutiérrez, J. J. & 1501 \\
\hline Angulo, Ignacio & 327,351 \\
\hline Anido Rifon, Luis & 1683 \\
\hline Anterrieu, Eric & 549 \\
\hline Antón, Miriam & 1295,1307 \\
\hline Añino, María Magdalena & 1385 \\
\hline Aparicio, Francisco & 1493 \\
\hline Aracil, Rafael & 967 \\
\hline Aranzadi, Pedro & 1039 \\
\hline Arcega Solsona, F. & $99,1173,1441$ \\
\hline Argüelles, Irina & 929 \\
\hline Argyriou, Vasileios & 743 \\
\hline Arias, Manuel & $717,1225,1779$ \\
\hline Ariol, Guillaume & 1853 \\
\hline Arredondo, Belen & 105 \\
\hline Arriaga, Jesús & $129,423,1039$ \\
\hline Arriero, Luis & 929 \\
\hline Aschenbrenner, Andreas & 943 \\
\hline Atienza, José M & 807 \\
\hline Atif, Yacine & 65 \\
\hline
\end{tabular}

\begin{tabular}{|c|c|}
\hline Auer, Michael E. & 585,1743 \\
\hline Aydin, Cansu Cigdem & 593 \\
\hline Aydin, Elif & 811 \\
\hline Baena, Carmen & 1217 \\
\hline Bailey, Philip & 145,731 \\
\hline Baladrón, Carlos & 77 \\
\hline Baldiris, Silvia Margarita & 473 \\
\hline Baley, Philip & 1409 \\
\hline Bárcena, Elena & 1137 \\
\hline Bargstädt, Hans Joachim & 639 \\
\hline Baron, Claude & 1851 \\
\hline Barone, Dante A. & 1147,1521 \\
\hline Barrero, Federico & 317 \\
\hline Barreto, Gilmar & 1943 \\
\hline Baumann, Meter & 523 \\
\hline Bellido, F.J. & 1451 \\
\hline Bellido, Luis & 1073 \\
\hline Belmekki, B. & 1937 \\
\hline Beltrán de Heredia, Á. & 1791 \\
\hline Benavente, César & 929 \\
\hline Benito, Manuel & 129 \\
\hline Benlloch Dualde, José V. & 671 \\
\hline Besbes, Riadh & 401 \\
\hline Bhave, Amit & 1085 \\
\hline Blanco Galán, Marcos & 1765 \\
\hline Blázquez Merino, Manuel & 1129,423 \\
\hline Boada, Imma & 973 \\
\hline Boehringer, David & 1091 \\
\hline Bonache, Jorge & 237 \\
\hline Borge, Marcela & 1587 \\
\hline Borromeo, Susana & 105 \\
\hline Bosch Estrada, José & 937 \\
\hline Boticario, Jesús G. & 797 \\
\hline Boto Giralda, Daniel & 1307 \\
\hline Boukachour, Hadoum & 725 \\
\hline Boulmalf, Mohammed & 685 \\
\hline Bragós, Ramón & 345,1403 \\
\hline Braumann, Andreas & 1085 \\
\hline Brito da Rocha, Claudio & 647,1057 \\
\hline Buendía, Félix & 671 \\
\hline Bulkowski, Alexander & 1667 \\
\hline Burguera, Antoni & 653 \\
\hline Burkhart, Helmar & 1075 \\
\hline Burr, Barbara & 853 \\
\hline Cabral, José Manuel & 159 \\
\hline Cabrera, Margarita & 429,1403 \\
\hline Cabrero Canosa, M. J. & 863 \\
\hline
\end{tabular}




\begin{tabular}{|c|c|c|c|}
\hline Caeiro Rodríguez, M. & $\begin{array}{l}99,869,1173 \\
1441,1683\end{array}$ & $\begin{array}{l}\text { Coble, Aaron R. } \\
\text { Cobo Benita, José Ramón }\end{array}$ & $\begin{array}{l}1085 \\
701\end{array}$ \\
\hline Cagiltay, Nergiz & $\begin{array}{l}243,811,879 \\
1633\end{array}$ & $\begin{array}{l}\text { Codreanu, Norocel } \\
\text { Coelho, Joao Vasco }\end{array}$ & $\begin{array}{l}113 \\
81\end{array}$ \\
\hline Callens, N. & 1701 & Colmenar, Antonio & 787 \\
\hline Calviño, Pablo & 631 & Colomer Farrarons, Jordi & 321 \\
\hline Camacho, David & 1131 & Comber, Oswald & 885 \\
\hline Camacho, José & 539 & Conde, Carlos & 631 \\
\hline Camacho, Michelle M. & 839 & Conejero, Alberto & 231,539 \\
\hline Camara, José A. & 631 & Corbalán, Montserrat & 1869 \\
\hline Cancelas, José A. & 1255 & Cordeiro, Joao & 1283 \\
\hline Candela, Santiago & 601 & Coronado, Sergio & 199 \\
\hline Canete Rebenaque, David & 1195 & Cortés, Francisco & 317 \\
\hline Cannella, Salvatore & 769 & Costa Freire, Joao & 173 \\
\hline Cano, Juan Carlos & 671 & Costa, Helder & 159 \\
\hline Canovas, Alejandro & 1273 & Costa, Monica & 1283 \\
\hline Capdevilla, Ramón & 1039 & Costa, Ricardo Jorge & 1359 \\
\hline Carmona Flores, Manuel & 937 & Costa, Rodrigo Garrett & 407 \\
\hline Carmona, Cristóbal J. & 983 & Cota, Manuel Pérez & 891 \\
\hline Carpio, José & $99,1173,1441$ & Cotfas, Daniel & 585,1641 \\
\hline Carrasco, Juan M. & 1165 & Cotfas, Petra & 585,1641 \\
\hline Carrasco, Ramon & 631 & Cousido, Carmen & 237 \\
\hline Carro, Belén & 77 & Crespo, Raquel & 1101,1233 \\
\hline Carro, German & 631 & & 1239 \\
\hline Carroll, John M. & 1461,1587 & Crusafon, Carlota & $\begin{array}{l}1239 \\
833\end{array}$ \\
\hline Carson, Stephen & 1659 & Cuadrado, Félix & $\begin{array}{l}850 \\
761\end{array}$ \\
\hline Carvalho, Adelson S. & 1147 & Cueva Carrión, S.P. & 121 \\
\hline Casany, Maria José & 1403 & Cussó, Roser & $\begin{array}{l}121 \\
833\end{array}$ \\
\hline Casquero, Oskar & 129 & Daniels, Mats & $\begin{array}{l}035 \\
1051\end{array}$ \\
\hline Castro, A. & 1701 & Dávila, Luis & 357 \\
\hline Castro, Manuel A. & $99,129,327$ & Day, Rally & 953 \\
\hline & $351,357,423$ & de Armas, Valentín & 1395 \\
\hline & $569,611,631$ & de Castro, Carlos & 1295 \\
\hline & $787,819,869$ & de la Fuente, Luis & 1101 \\
\hline & 907,1137 & de la Torre, Isabel & 1295,1307 \\
\hline & $1171,140 \%$ & de Vries, Pieter & 1065 \\
\hline & 1841,1731, & del Arco Fdez Cano, E. & 1265 \\
\hline & $\begin{array}{l}1829,1869, \\
1909\end{array}$ & del Blanco, Ángel & 1121 \\
\hline Cedazo León, Raquel & $\begin{array}{l}1909 \\
1367\end{array}$ & del Jesus, María José & 983 \\
\hline Chaiko, Yelena & 695 & Delgado Kloos, Carlos & $303,503,110$ \\
\hline Charlton, Terence James & $\begin{array}{l}093 \\
179\end{array}$ & & 1233,1239 \\
\hline Chatterjee, Arunangsu & 897 & & 1247 \\
\hline Chavez, Igor & 631 & Delgado, José Luis & 1137 \\
\hline Chen, Chun Yu & 575 & DeLong, Kimberly & 145,1409 \\
\hline Chen, John C. & 381 & Derntl, Michael & 1237 \\
\hline Chicaiza, Janneth & 111,1513 & Devlin, Marie & 179,271 \\
\hline Chuang, Sheng Hsiung & 457 & Dias, Octavio Páscoa & 561 \\
\hline Ciampi, Melany & 647,1057 & Dias, Pedro Miguel & 1007 \\
\hline Ciancimino, Elena & 769 & Díaz Lantada, Andres & 1483 \\
\hline
\end{tabular}




$\begin{array}{ll}\text { Díaz Orueta, Gabriel } & 1909 \\ \text { Díaz, Francisco Javier } & 1295 \\ \text { Díaz, Gabriel } & 99,819,907, \\ & 1173,1441, \\ & 1731 \\ \text { Díaz, Pablo } & 1739 \\ \text { Díaz, Paloma } & 1931 \\ \text { Díaz Pernas, Francisco J. } & 1307 \\ \text { Díez, David } & 1931 \\ \text { Díez, José Fernando } & 1295,1307 \\ \text { Dilhac, Jean Marie } & 1853 \\ \text { Domingo, Rosario } & 1859 \\ \text { Domínguez, Eugenio } & 1165 \\ \text { Domínguez, Manuel } & 99,1173,1441 \\ \text { Donnelly, Anne } & 1843 \\ \text { Dopico, Alberto } & 631 \\ \text { Dos Santos, Fabio R. } & 731 \\ \text { Drlik, Martin } & 1899 \\ \text { Drummond, Sarah } & 179 \\ \text { Duda, Andrzej } & 1667 \\ \text { Dueñas, Juan C. } & 761 \\ \text { Durán Escribano, Pilar } & 1649 \\ \text { Duran, Alfonso } & 1923 \\ \text { Echavarri Otero, Javier } & 1483 \\ \text { Elices, Manuel } & 807 \\ \text { Emmna, F. } & 1701 \\ \text { Enuma, Clara } & 1875 \\ \text { Eppes, Tom A. } & 845,1425 \\ \text { Escovedo, Tatiana } & 1205 \\ \text { Exposito, Ernesto } & 1819 \\ \text { F. Linera, Francisco } & 1779 \\ \text { Fabregat, Ramón } & 473,973 \\ \text { Faccioni Filho, Mauro } & 677 \\ \text { Falcone, Francisco } & 99,1173,1441 \\ \text { Falsetti, Carla } & 1527 \\ \text { Fayolle, Jacques } & 1937 \\ \text { Fernández, Pilar } & 755 \\ \text { Fernández Manjón, B. } & 775,1121 \\ \text { Fernández Miaja, Pablo } & 1225 \\ \text { Fernández Mostaza, Mã. } & 833 \\ \text { Fernández Panadero, C. } & 503 \\ \text { Fernández Nieto, G. M. } & 1289 \\ \text { Fernández Pantoja, Mario } & 329 \\ \text { Fernández Rodrigues, J.A } & 451 \\ \text { Fernao Pires, Vitor } & 561 \\ \text { Ferre, Manuel } & 967 \\ \text { Ferreira, André Luis A. } & 407 \\ \text { Figueiredo, António Dias } & 1317 \\ \text { Figueiredo, José } & 531 \\ & \\ & \end{array}$

$\begin{array}{ll}\text { Figuera, Carlos } & 1265 \\ \text { Fitzgerald, Alan } & 389\end{array}$

$\begin{array}{ll}\text { Fitzgerald, Alan } & 389 \\ \text { Flores Arias, José Maria } 145\end{array}$

Florián Gaviria, Beatriz 473

Fontenla González, Jorge 869

Fontes, Ricardo $\quad 1283$

Forward, Mary Lou $\quad 1659$

Fourniols, Jean Yves 1853

Fraile, Ruben 929

Francisco, Jesús $\quad 1541$

Franco Neto, Moacyr 677

Franquelo, Leopoldo G. 1165

Freudenthal, Eric $\quad 663,991$

Friesel, Anna 1327

Fritzson, Peter 1081

Fuhrmann, Thomas $\quad 313$

Gadwal, Apeksha $\quad 287$

Galán, Ramón $\quad 465$

Galán, Santos $\quad 1711$

Galeano, Katherine J. 211

Galeone, P. $\quad 1701$

Galindo, Ernest 1493

Galinho, Thierry 725

Galkina, Alina $\quad 369$

Gámez, Juan Carlos 1189

Ganoe, Craig $\quad 1587$

Garbi Zutin, Danilo 1743

García, Ángel $\quad 967$

García, Carmelo R. $\quad 601$

García, Eduardo $\quad 1453$

García, Enrique $\quad 1295$

García, Miguel 1273

García, Pablo 1255

García Borrás, Patricia 465

García Campos, Rafael 91

García Doval, Fátima Ma 1785

García Martín, Javier 1575

García Pastor, Fabian 1751

García Robledo, Pablo 465

García Ruiz, Francisco 329

García Sánchez, Manuel 1299

García Sevilla, Francisco 1869

García Zubia, Javier $\quad 327,351$

Garofano, Francesc $\quad 345$

Gay Fernández, José A. 1299

Ghercioiu, Marius $\quad 585$

Gil, Charo 631, 1731

Gil, Marisa 833

Gil García, José Miguel 365

Gillet, Denis P. 897 


\begin{tabular}{|c|c|c|c|}
\hline Giraldo, Esmeralda & 1923 & Hasna, Abdallah & 1603 \\
\hline Gironella, Xavier & 1403 & Heiß, Hans Ulrich & 639 \\
\hline Glew, William & 389 & Heo, Jun Haeng & 295 \\
\hline Godino Llorente, Juan I. & 929 & Hercog, Darko & 959 \\
\hline Göhner, Peter & 853 & Herms Berenguer, Atila & 937 \\
\hline Gomes, Marcelo Carboni & 1521 & Hernández, Luis & 967 \\
\hline Gomez Cama, José M. & 937 & Hernández, Roberto & 611 \\
\hline Gómez Tornero, José L. & 1195 & Hernández, Rocael & 611 \\
\hline Gómez, Iñigo Cuiñas & 1299 & Hernández, Unai & 327,351 \\
\hline Gómez, Isabel M. & 1217 & Hernández, Filmar & 237 \\
\hline Gomis, Oriol & 1401 & Hernando, Marta & 1779 \\
\hline González, Juan C. & 929 & Hernán Losada, Isidoro & 1557 \\
\hline Gonzalez, Julio Jorge & 1875 & Herrero, David & 1541 \\
\hline González, María Jesús & 1791 & Hinojo, José María & 317 \\
\hline González, Miguel & 1501 & Hoffmann, Michael H.W. & 639 \\
\hline González, Oscar & 543 & Horwath, Karla Chagas & 1521 \\
\hline González, Pedro & 983 & Hoyos Gomez, Guillermo & 281 \\
\hline González, Victor M. & 1255 & Huang, Hsin Hsiung & 457 \\
\hline González Aragón, M.1 & 1813 & Huang, Jheng Yu & 457 \\
\hline González Barahona, J. M. & 1129 & Huhtamäki, Jukka & 137 \\
\hline González García, S. & 329 & Hvorecky, Jozef & 1899 \\
\hline González González, C.S. & 1479 & Ibáñez, M ${ }^{\mathrm{a}}$ Blanca & 1101 \\
\hline González Lamar, Diego & $223,717,1779$ & Iriguchi, Norio & 153 \\
\hline González Ortega, David & 1307 & Irurzun, Jaime & 327,351 \\
\hline González Téllez, Alberto & 415 & Islam, Syed Zahidul & 1159 \\
\hline Gora, Wojtek & 1893 & Izu, Cruz & 997 \\
\hline Gorce, Philippe & 401 & Jacobi, Jane & 1843 \\
\hline Grabowski, Jens & 943 & Jara Roa, Dunia Inés & 217 \\
\hline Grande, Ana & 543 & Jeschke, Sabina & $825,853,1893$ \\
\hline Gravier, Christophe & 1937 & Jezernik Karen & $\begin{array}{l}825,850,1895 \\
959\end{array}$ \\
\hline Gregorio, Robles & 1129 & $\begin{array}{l}\text { Jezernik, Karen } \\
\text { Jiang, Hao }\end{array}$ & $\begin{array}{l}959 \\
1587\end{array}$ \\
\hline Grieu, Jean & 725 & $\begin{array}{l}\text { Jiang, Hao } \\
\text { Jiang, Jin }\end{array}$ & $\begin{array}{l}158 / \\
481\end{array}$ \\
\hline Guasch, Aleix & 345 & $\begin{array}{l}\text { Jang, Jin } \\
\text { Jimenez Trillo Juan }\end{array}$ & $\begin{array}{l}481 \\
237\end{array}$ \\
\hline Guerreiro, Pedro & 263 & $\begin{array}{l}\text { Jimenez Trillo, Juan } \\
\text { Jofre Lluis }\end{array}$ & $\begin{array}{l}231 \\
1761\end{array}$ \\
\hline Guerrero, José & 653 & $\begin{array}{l}\text { JoIre, Liuis } \\
\text { Jonosawat Ninat }\end{array}$ & $\begin{array}{l}1 / 01 \\
1533\end{array}$ \\
\hline Guerrero Curieses, A. & 783 & $\begin{array}{l}\text { Jongsawat, Nipat } \\
\text { Jordá Albiñana, Begoña }\end{array}$ & $\begin{array}{l}1535 \\
1509\end{array}$ \\
\hline Guetl, Christian & 493,731 & Jordana, Joséph & 395 \\
\hline Guevara Bolaños, Juan C. & 1289,1951 & Juan, Jordi & 231 \\
\hline Guggisberg, Martin & 1075 & Jucovschi, Constantin & 523 \\
\hline Guinea, Gustavo V & 807 & Jurado, Francisco & $99,1173,1441$ \\
\hline Günther, Markus & 191 & Kalman, Tibor & 943 \\
\hline Gutiérrez Reina, Daniel & 317 & Kamal Eddine, El Kadiri & 1801 \\
\hline \multirow[t]{2}{*}{ Gutiérrez Rojas, Israel } & 303,1101 & Kang, So Yeon & 295 \\
\hline & 1233,1239 & Kenyon, Tony & 953 \\
\hline Gutiérrez, Juana M. & 237,929 & Khachadorian, Sevak & 1065 \\
\hline Gutiérrez Pérez, David & 1265 & Kicken, Wendy & 819 \\
\hline Gyalog, Tibor & 1075 & Kiesling, Elmar & 191 \\
\hline Hampe, Manfred & 639 & Kita, Toshihiro & 153 \\
\hline Hardison, James & 145,1409 & Klein, Lawrence Zordam & 677 \\
\hline Harward, Judson & $145,731,1409$ & Knipping, Lars & 825 \\
\hline
\end{tabular}




\begin{tabular}{|c|c|}
\hline Kohse, Gordon & 145 \\
\hline Komlenov, Zivana & 885 \\
\hline Kondabathini, Vishal & 751 \\
\hline Koper, Rob & 1211 \\
\hline Köppel, Grit & 825 \\
\hline Kraft, Markus & 1087 \\
\hline Ktoridou, Despo & 1807 \\
\hline Kubota, Shinichiro & 153 \\
\hline Kunicina, Nadezhda & 369,695 \\
\hline Labrador, Manuel & 237 \\
\hline Lach, Gerald & 1893 \\
\hline Lafont Morgado, Pilar & 1483 \\
\hline Lafuente, Guillermo & 631 \\
\hline Lakas, Abderrahmane & 685 \\
\hline Landaluce, Ariana & 129 \\
\hline Lauer, Gerhard & 943 \\
\hline Law, Effie L C. & 897 \\
\hline Layton, Richard A. & 839 \\
\hline Lázaro, Carlos & 1557 \\
\hline Lecroq, Florence & 725 \\
\hline Lee, Chyi Shyong & 457 \\
\hline Lee, Joosung & 295 \\
\hline Leo, Tommaso & 1527 \\
\hline Leon, José I. & 1165 \\
\hline Leony, Derick & 1101,1239 \\
\hline LI, Yamin & 225 \\
\hline Lin, Shean Huei & 575 \\
\hline Linan, M. & 1451 \\
\hline Ling, Jonathan Geoffrey & 389 \\
\hline Linsey, Julie & 287 \\
\hline Llamas Nistal, Martín & $\begin{array}{l}99,869,1173, \\
1441\end{array}$ \\
\hline Llamosa Villalba, R. & 1957 \\
\hline Lloret, Jaime & 1273 \\
\hline López Campos, Mónica & 769 \\
\hline López de Miguel, Manuel & 1937 \\
\hline López Zamarrón, Diego & 1367 \\
\hline Lopez, Eugenio & 631 \\
\hline López, Jorge & $129,1111,1513$ \\
\hline López, Miguel & 1295,1307 \\
\hline López Puche, Pilar & 1575 \\
\hline Lord, Susan & 381,839 \\
\hline Lorente Leal, Alberto & 451 \\
\hline Lorenzo, Emilio J. & 1137 \\
\hline Lozano Tello, Adolfo & 1765 \\
\hline Lübbe, Jan & 1893 \\
\hline Luengas, Lely Adriana & 211,1951 \\
\hline Machado, Felipe & 105 \\
\hline Machado, José & 1277 \\
\hline
\end{tabular}

\begin{tabular}{|c|c|}
\hline Madeira, Rui Neves & $561,1013,1673$ \\
\hline Madrigal, David & 1541 \\
\hline Magenheim, Johannes & 513 \\
\hline Magoc, Tanja & 991 \\
\hline Maier, Christian & 1743 \\
\hline Maillet, Catherine & 71 \\
\hline Maiti, Ananda & 1351 \\
\hline Malpica, Norberto & 105 \\
\hline Mandado, Enrique & 755 \\
\hline Manganello, Flavio & 1527 \\
\hline Manso, António & 1007 \\
\hline Manzanares Bolea, R. & 1419 \\
\hline Marchiori, Eugenio J. & 1121 \\
\hline Marcos, Jorge & 445 \\
\hline Mariño, José B. & 1403 \\
\hline Marques, Célio & 1007 \\
\hline Marquez, Juan J. & 709 \\
\hline Marshall, Lindsay & 179 \\
\hline Martín, Estefanía & 1557 \\
\hline Martín, Sergio & $\begin{array}{l}569,631,787, \\
819,1137\end{array}$ \\
\hline Martínez Bejarano, R. & 1419 \\
\hline Martínez Mateo, Jesús & 913 \\
\hline Martínez Muneta, M L. & 709 \\
\hline Martínez, David & 1791 \\
\hline Martínez, Oscar & 1111 \\
\hline Martínez Calero, José D. & 1869 \\
\hline Martínez Mediano, C. & 819 \\
\hline Martínez Zarzuela, Mario & 1307 \\
\hline Martinich, Leslie P & 1551 \\
\hline Martins, Joao & 561 \\
\hline Martins, Scheila Wesley & 1317 \\
\hline Más, Jorge & 231 \\
\hline Mateos, Verónica & 1071 \\
\hline Matsuba, Ryuichi & 153 \\
\hline Mayorga, José I. & 1139 \\
\hline McIntyre, Michael & 1715 \\
\hline Md Yusoff, Yuzainee & 921 \\
\hline Medeiros, Claudia Bauzer & 523 \\
\hline Medrano, Carlos & 1869 \\
\hline Melo, Rubens & 1205 \\
\hline Mena Mena, Alexis & 281 \\
\hline Mendes, António José N. & 1317 \\
\hline Méndez, Sergio & 1957 \\
\hline Meriläinen, Joonas & 137 \\
\hline Merkuryev, Yuri & 1721 \\
\hline Mestres Sugrañes, Albert & 429 \\
\hline Metrolho, José Carlos & 1283 \\
\hline Meyer, Jörg & 943 \\
\hline Miaja, Pablo F & 717 \\
\hline
\end{tabular}




\begin{tabular}{|c|c|}
\hline Migita, Masahiro & 153 \\
\hline Miguel, Paulo Victor O. & 1943 \\
\hline Miilumäki, Thumas & 137 \\
\hline Mikami, Akane & 185 \\
\hline Milanovic, Ivana & 845 \\
\hline Mileva, Nevena & 819 \\
\hline Minguet, Jesús M. & 907 \\
\hline Miribel Català, Pedro L. & 321 \\
\hline Mißler, Rüdiger & 205 \\
\hline Mitchell, John & 953 \\
\hline Mitrofanov, Oleg & 953 \\
\hline Mohamed, Azah & 921 \\
\hline Mohamed, Khaldi & 1801 \\
\hline Moncef BenKhélifa, M. & 401 \\
\hline Monteiro, A. Caetano & 1277 \\
\hline Montero, Eduardo & 1791 \\
\hline Montero, Juan M & 451 \\
\hline Montero, Susana & 1931 \\
\hline Morales, Asunción & 1395 \\
\hline Moreno Ger, Pablo & 1121 \\
\hline Moreno Munoz, A. & 1451 \\
\hline Morgado, Eduardo & 1265 \\
\hline Morillas, Samuel & 231,539 \\
\hline Moss, Keith Edward & 1883 \\
\hline Motschnig, Renate & 885 \\
\hline Muhamad, Norhamidi & 921 \\
\hline Mulder, Ingrid & 1433 \\
\hline Müller, Florian & 1075 \\
\hline Müller, Gerhard & 639 \\
\hline Munk, Michal & 1899 \\
\hline Muñoz Guijosa, Juan M. & 1483 \\
\hline Muñoz García, Julio & 1483 \\
\hline Muñoz Hernández, S. & 913 \\
\hline Muñoz Merino, Pedro & 1101 \\
\hline Muñoz Organero, Mario & 1101 \\
\hline Muñoz Sanz, José Luis & 1483 \\
\hline Muñoz, Javier & 1739 \\
\hline Muñoz Fernández, Isabel & 1575 \\
\hline Mur, Francisco & $99,1173,1441$ \\
\hline Murari, Carlos Alberto F & 1943 \\
\hline Murillo, Gloria & 631 \\
\hline Musashi, Yasuo & 153 \\
\hline Mustapha, Ramlee & 921 \\
\hline Nadeem, Danish & 1211 \\
\hline Nagai, Takayuki & 153 \\
\hline Najima, Daoudi & 1693 \\
\hline Najjar, Jad & 1239 \\
\hline Nakamura, Taichi & 185 \\
\hline Nakano, Hiroshi & 153,165 \\
\hline Natho, Nicole Martina & 825 \\
\hline
\end{tabular}

\begin{tabular}{|c|c|}
\hline Nawarecki, Edward & 1667 \\
\hline Nelles, Wolfgang & 513 \\
\hline Nesterova, Elena & 487 \\
\hline Neumann, Susanne & 1239 \\
\hline Niederstätter, Michael & 1743 \\
\hline Nishihara, Akinori & 1031 \\
\hline Nottis, Katharyn & 381 \\
\hline Oberhuemer, Petra & 1239 \\
\hline Ogrey, Alexandria N. & 663 \\
\hline Ohland, Mathew W. & 839 \\
\hline Olivares, Joaquín & 1189 \\
\hline Oliveira, Joaquim & 1153 \\
\hline Oliver, Joan & 1343 \\
\hline Oliver, Sonia & 1835 \\
\hline Omar, Mohd Zaidi & 921 \\
\hline Ordieres Meré, Joaquín & 701 \\
\hline Orduña, Pablo & 327,351 \\
\hline Ortiz Marcos, Isabel & 701 \\
\hline Osés, David & 929 \\
\hline Ostrovsky, Yakov & 145 \\
\hline Pacios Álvarez, Antonia & 701 \\
\hline Page, Helen & 1701 \\
\hline Palmero, Javier & 237 \\
\hline Palomares, José Manuel & 1189 \\
\hline Pardo, Abelardo & 1101,1247 \\
\hline Paredero, Ruben A. & 631 \\
\hline París Regueiro, $\mathrm{M}^{\mathrm{a}} \mathrm{T}$. & 863 \\
\hline Parra, M. Pilar & 1217 \\
\hline Pasman, Gert & 1433 \\
\hline Pastor, Rafael & $\begin{array}{l}99,611,1173, \\
1441\end{array}$ \\
\hline Pastor, Vicente & 1909 \\
\hline Patlins, Antons & 695 \\
\hline Payeras, Magdalena & 653 \\
\hline Pears, Arnold Neville & 1051 \\
\hline Peire, Juan & 569 \\
\hline Pelayo, Sofia & 1277 \\
\hline Penn, Martin & 389 \\
\hline Perassi, Marisol & 1385 \\
\hline Pereda, José Antonio & 543 \\
\hline Pérez Gutiérrez, Byron A. & 281 \\
\hline Pérez López, Serafín A. & 445 \\
\hline Pérez Molina, Clara & 1139 \\
\hline Pérez, Jesús M. & 709 \\
\hline Pérez, José Philippe & 549 \\
\hline Pérez, Marimar & 1403 \\
\hline Pérez Gama, Alfonso & 281 \\
\hline Pérez Martínez, Jorge E. & 1575 \\
\hline Pérez Rey, David & 913 \\
\hline Pérez Rodriguez, I & 1683 \\
\hline
\end{tabular}




\begin{tabular}{|c|c|c|c|}
\hline Person,Patrick & 725 & Rings, Thomas & 943 \\
\hline Pescador, Fernando & 129,423 & Rio, Miguel & 953 \\
\hline Petschik, Grit & 825 & RioPérez, Nuria & 819 \\
\hline Pfeiffer, Olivier Frédéric & 1893 & Rius, Juan Manuel & 1403 \\
\hline Phillips, Chris & 271 & Rivera, Adexe & 601 \\
\hline Piedra, Nelson & $129,1111,1513$ & Rocha, José Gerardo & 159 \\
\hline Pierce McMahon, Joana & 1649 & Rodrigo, Covadonga & 1137 \\
\hline Pinto, Enrique & 967 & Rodríguez Artacho, M. & 217,1137 \\
\hline Pires, Jorge Manuel & 891 & Rodriguez Hernández, A. & 621 \\
\hline Pistoia, Antonio & 1527 & Rodríguez Morales, G. & 121 \\
\hline \multirow[t]{2}{*}{ Plaza, Inmaculada } & $99,1173,1441$ & Rodríguez, Alberto & $717,1225,1779$ \\
\hline & 1869 & Rodríguez, Miguel & 717,1225 \\
\hline Poch, Jordi & 973 & Rodríguez González, Ana & 783 \\
\hline Pohjolainen, Seppo & 137 & Rodriguez Losada, Diego & 553 \\
\hline Polito, Catherine & 1549 & Rodríguez Navas, G & 653 \\
\hline Ponsa, Pedro & 1335,1835 & Rodríguez Santiago, N. & 329 \\
\hline Pop, Adrian & 1081 & Rojas Sola, José Ignacio & 1509 \\
\hline Porta, Marcela & 71 & Rojko, Andreja & 959 \\
\hline Portillo, Ramon & 1165 & Romá, Miguel & 1455 \\
\hline Pou Felix, Joséph & 1869 & Romanovs, Andrejs & 1721 \\
\hline Pousada Carballo, J.M ${ }^{a}$ & 1785 & Romans, Ed & 953 \\
\hline Prados, Ferran & 973 & Romero, Audrey & 121,1513 \\
\hline Premchaiswadi, Wichian & 1533 & Romero, Carmen & 1217,1835 \\
\hline Prince, Michael & 381 & Romero, Cristóbal & 983 \\
\hline Pueo, Basilio & 1455 & Romero, Gregorio & 709 \\
\hline Puig i Bosch, Jordi & 91 & Romo, Jesús & 129 \\
\hline Pulido, Estrella & 1131 & Ros, Salvador & 611,1137 \\
\hline Qian, Jinwu & 225,481 & Rosson, Mary Beth & 1461 \\
\hline Quadt, Arnulf & 943 & Ruiz, Elena & 907 \\
\hline Quesada Pereira, F. D. & 1195 & Ruiz, Jonathan & 327 \\
\hline Quesada, Jerónimo & 365,1829 & Ruiz de Garibay, J. & 351 \\
\hline Quintáns, Camilo & 445 & Sáenz, Mauricio & 1041 \\
\hline Rachida, Ajhoun & 1693 & Sagi Vela, Javier & 1039 \\
\hline Ramírez, F. Javier & 1859 & Sainz, Beatriz & 1295 \\
\hline Ramis, Jaume & 653 & Sainz, José Antonio & 365 \\
\hline Ramos, Daniel & 1181 & Sainz de Abajo, Beatriz & 1307 \\
\hline Raud, Zoja & 1613 & Salaverría, Angel & 755 \\
\hline Read, Tim M. & 611,1137 & Sallier, René & 205 \\
\hline Reis, Cristina & 1277 & Salvado, José & 1153 \\
\hline Rela, Mário Zenha & 1359 & Samoila, Cornel & 585,1641 \\
\hline Renaud, Cyril & 953 & San Cristóbal, Elio & $327,351,357$ \\
\hline Requena Carrión, Jesús & 783 & & $631,819,869$ \\
\hline Ressel, Wolfram & 853 & & 1409 \\
\hline Rhode, Thomas & 513 & San Segundo, Pablo & 553 \\
\hline Ribickis, Leonids & 369,695 & Sanborn, Jennifer & 845 \\
\hline Richter, Christoph & 1595 & Sánchez, Alicia & 631 \\
\hline Richter, Thomas & 1091 & Sánchez, Antonio & 77 \\
\hline Rico, Mariano & 1131 & Sànchez, Francesc J. & 395 \\
\hline Rincón, David Andrés & 211 & Sánchez, Francisco & $99,1173,1441$ \\
\hline
\end{tabular}




\begin{tabular}{|c|c|c|c|}
\hline Sánchez, Jaime, & 1041 & Stoyanov, Slavi & 819,1211 \\
\hline Sánchez, José Ángel & 99,1441 & Stummer, Christian & 191 \\
\hline Sánchez, Juan A. & 1165 & Su, Juing Huei & 457 \\
\hline Sánchez Alejo, F.J. & 1493 & Sugitani, Kenichi & 153 \\
\hline Sánchez Moreno, F.M. & 1367 & Sustelo, Maria F. & 263 \\
\hline Sánchez Ortiz, José A. & 1173 & Takashima, Akio & 185 \\
\hline Sánchez Reillo, Raul & 1419 & Tchoumatchenko, V.P. & 1597 \\
\hline Sánchez Terrones, B. & 345 & Tebest, Teemo & 137 \\
\hline Sancho, Pilar & 775 & Tetour, Yvonne & 1091 \\
\hline Santana, Iván & 967 & Thomsen, Benn & 953 \\
\hline Sapena, Almanzor & 539 & Thomsen, Christian & 1065 \\
\hline Sarango Lapo, Celia P. & 217 & Tirkeş, Güzin & 593 \\
\hline Savory, Seb & 953 & Tobajas, Félix B. & 1395 \\
\hline Schaper, Niclas & 513 & Tokdemir, Gul & 243 \\
\hline Scheel, Harald & 1065 & Toledo, Ricardo & 1343 \\
\hline Schlicht, Wolfgang & 853 & Torabzadeh Tari, M. & 1081 \\
\hline Schmitt, Heike & 639 & Toral Marín, Sergio L. & 317 \\
\hline Schröder, Christian & 825 & Torrente, Javier & 1121 \\
\hline Schubert, Sigrid & 513 & Totschnig, Michael & 1239 \\
\hline Schütze, Andreas & 205 & Tovar, Edmundo & $99,129,423$, \\
\hline Seabra, Eurico & 1277 & & 1111,1173 \\
\hline Sebastián y Zúñiga, J.M. & 1367 & & 1441,1513 \\
\hline Sebastián, Javier & 717 & & 1657 \\
\hline Sebastián, Miguel Ángel & 1813,1859 & Tseng, Ya Fen & 1391 \\
\hline Sebastián, Rafael & 1829 & Tungkasthan, Anucha & 1533 \\
\hline Semmar, Yasser & 685 & Tzanova, Slavka & 113,819 \\
\hline Sendra, Sandra & 1273 & Ubeda Mansilla, Paloma & 1649 \\
\hline Sevaslidou, Maria & 743 & Ulloa, Ricardo & 487 \\
\hline Sevilla Hurtado, Lorenzo & 1813 & Urquiza Fuentes, Jaime & 1019 \\
\hline Shih, Chien Chou & 575 & Ursutiu, Doru & 585,1641 \\
\hline Shih, Yen Hua & 575 & Usagawa, Tsuyoshi & 153 \\
\hline Shuaib, Khaled & 685 & Val, José Luis del & 1753 \\
\hline Sierra Alonso, Almudena & 1575 & Valderrama, Elena & 1343 \\
\hline Silius, Kirsi & 137 & Valdiviezo Díaz, P.M. & 217 \\
\hline Silva, Bruno & 1011 & Valencia, Manuel & 1217 \\
\hline Sim, Tze Ying & 1565 & Vallejo, Enrique & 1453 \\
\hline Simões Piedade, Moisés & 173 & Vaquero, Joaquin & 105 \\
\hline Simon, Bernd & 1239 & Vara, Alfonso & 907 \\
\hline Sivianes, Francisco & 1217 & Vargas Berzosa, Carlos & 429 \\
\hline Smallbone, Andrew & 1085 & Vargas Berzosa, F. & 429 \\
\hline Soler, Joséph & 973 & Vasileva, Tania Krumova & 1597 \\
\hline Somacarrera, Maria Luisa & 1923 & Vázquez Alejos, Ana & 1299 \\
\hline Soshko, Oksana & 1721 & Vázquez Martínez, Juan & 1419 \\
\hline Soto Merino, Juan C. & 253 & Vazquez, Sergio & 1165 \\
\hline Soto, José Manuel & 1189 & Vegas, Angel & 543 \\
\hline Souhaib, Aammou & 1801 & Velázquez Iturbide, Á. & 1019 \\
\hline Stechert, Peer & 513 & Velez, Javier & 1137 \\
\hline Stefan, Alexandru & 1641 & Vendrell, Eduardo & 231 \\
\hline Stefanou, Candice & 381 & Ventura, Sebastián & 983 \\
\hline Stolk, Jonathan & 381 & Ventura Traveset, J. & 1701 \\
\hline
\end{tabular}


Verd, Jaime 653

Vez Jeremías, José M. 1785

Viezens, Fred 943

Vilanova, Ramón $\quad 1335,1835$

Villagrá., Víctor $\quad 1071$

Villena Román, Julio 503

Vinjarapu, Sai Krishna D. 751

Viswanathan, Vimal K. 287

Vodovozov, Valery 1613

Vogel Heuser, Birgit 1565

Waigandt, Diana M. 1385

Walker, Roger $\quad 1701$

Wang, Tsung Li 1391

Wang, Xiaojing 225

Wannous, Muhammad 165

Watson, Roger $\quad 1085$

Wenk, Bruno 435

Williams, Bill $\quad 531$

Wilson, Stacy $\quad 1715$

Winzker, Marco $\quad 375$

Wyne, Mudasser F. $\quad 1623,1921$

Yaman, Seniz 1633

Zafeiriou, Stefanos $\quad 743$

Zaharim, Azami 921

Zaman, Muhammad 1047

Zampunieris, Denis 199

Zaro, Milton Antônio $\quad 407,1145$

Zayas Gómez, David 1501

Zhao, Ling 481

Zhiravecka, Anastasija 695

Zimmermann, Martin 1773

Zorn, Erhard $\quad 825$ 


\section{Contributions Index}

"e-Adventure" - Introducing Educational Games in the Learning Process

A competitive collaborative learning experience in chemical plant design

A Concept Map Approach for Introduction to Computer Engineering Course

A context for programming learning based on research communities

A Knowledge based analytical model of propaedeutic cycles for Higher Education in Colombia: Linking Media Education to Higher Education in Colombia.

A Learning Approach Based on Robotics in Computer Science and Computer Engineering

A Middleware for the Integration of Third-party Learning Tools in SOAbased Learning Management Systems

A New Competencies Assessment Data Model

A new Systemic Methodology for Lab Learning based on a Cooperative Learning Project

A Practical Electronic Instrumentation Course for Engineering Students

A Project-Based Learning Approach to Teaching Power Electronics

A Project-Oriented Integral Curriculum on Electronics for

Telecommunication Engineers

A Proposal for the Evaluation of Final Year Projects in a Competence-based Learning Framework

A Remote Laboratory to Promote the Interaction between University and Secondary Education

A review of electronic engineering design free software tools

A simulation software for sequential control

A Student-Centered Collaborative Learning Environment for Developing Communication Skills in Engineering Education

A Study and a Proposal of a Collaborative and Competitive Learning Methodology

A system to manage the alocation of MSc Dissertations at University of Minho

A Systems Theory Perspective of Electronics in Engineering Education

A Tablet PC-Based Teaching Approach using Conceptual Maps

A Tool to Reveal the Students Work Activity Along an Academic Period 
Action Research: A Way to Generate New Approaches to Teaching

Mathematics in Bioengineering

Active Learning in Power Electronics

Active Learning in Telecommunication Engineering: A case study

Adaptation in a PoEML-based E-learning Platform

Adapting the Telecommunication Engineering curriculum to the EEES: a

project based learning tied to several subjects

Adaptive Ecology M-Learning for National Park Based on Scaffolding

Theory

Adaptive hypermedia systems for e-learning

1801

Adjunt Enterprise Professors in the European Higher Education Area

Aligning Assessment with Learning Outcomes in Outcome-based Education

An adaptive Multi-Agent based Architecture for Engineering Education

An application-case for derivative learning: optimization in colour image

filtering

An approach for Description of Open Educational Resources based on semantic technologies

An enterprise e-learning solution: The UNED practical case in the EHEA

An Experience in Cooperative Learning Developing a Real Aerospacial Project

An Experience of a Multidisciplinary Activity in a Biomedical Engineering Master Degree

An integrated system as a tool for complex technology learning

An Interdisciplinary Practical Course on the Application of Grid Computing

An Undergraduate Microwave and RF Low-Profile Laboratory

Analysis of the results of four years of research and application of a student-

Analyzing self-reflection by Computer Science students to identify bad study habits

Applying a methodology for collaborative assesment in learning groups Innovation \& Technology Course: Evidence from the University of Nicosia Approach to Teaching Communications Systems by Collaborative Learning. Student Perceptions in the application of Problem-Based Learning.

Assessing Assessment Formats: The Current Picture

Assessing Competency in Undergraduate Software Engineering Teams

Assessment of learning activities in discussion forums online

Assessment of the learning competence of mathematics for first-years of the

Computer Science degree

Attracting Student Vocations into Engineering Careers. EnginyCAT:

Catalonia Promotional and Prospective Plan

Attracting, Retaining, and Preparing a Diverse Academic Engineering Workforce 
Authoring Environment for E-learning Production Based on Independent

XML Formats

Authoring Learning Contents, Assessments and Outcomes in an Integrated

Way

Automatic Guidance Tools for Enhancing the Educative Experience in Non-

Immersive Virtual Worlds Preliminary results from project V-LeaF

Bringing the everyday life into engineering education

Business and Management Competency of Engineers: Curriculum and

Assessment

Challenges in an Emerging Country: A Digital Divide Case Using Robotics

Collaborative Subjects for Embedded Systems Learning in the EHEA Frame:

A Practical Approach

Competence certification as a driver for professional development: A IT-

related exploratory case-study

Competencies for Informatics Systems and Modeling. Results of Qualitative

Content Analysis of Expert Interviews

Competency-Based Pedagogical Wrapping

Computation for Science and Engineering

Consequences of the Declining Interest in Engineering Studies in Europe

Continuous Proactivity in Learning Management Systems

Cooperative assessment in the hands on skills of computer networks subjects

Cooperative Learning vs. Project Based Learning: a practical case

Cooperative work and continuous assessment in an Electronic Systems

laboratory course in a Telecommunication Engineering degree

Course design approaches for the EHEA. Scaling up from pilots.

1923

Current Issues With Assessment Formats and Interoperability

Cybertech: Robotic Competition and Subject

Database Teaching tools

1883

Deep Drawing Tool for E-learning

Delievering authentic experiences for engineering students and professionals

Deployment of Remote Experiments: The OnPReX course at the TU Berlin

Design lab work in telecom

Design of an Educational Oscilloscope

Design of an Introductory Networking subject in advance of the European

Higher Education Area: Challenges, experiences and open issues

Developing an Optical Spectrum Analyzer

Developing and Evaluating a Game-Based Project Management Learning

Platform

Developing Global Teamwork Skills: The Runestone Project 
Development of a Small Radio Telescope at the Technical University of

Development of a Wiimote-based gesture recognizer in a microprocessor laboratory course

Development of the OCW Consortium

Developping of Low Cost Capacitive Sensors for Laboratory Classes

Didactic videos about basic concepts on alternating current circuits

Directions in Quality

Distance Practices in Subjects of Automatic Control

Distributed Collaborative Homeworks: Learning Activity Management and

Dual Instructional Support Materials for introductory object-oriented programming: classes vs. objects

Dynamic Virtual Environment for multiple Physics Experiments in Higher Education

Easily Integrable platform for the deployment of a remote laboratory for microcontrollers

Educational Computer Tool for Visualizing and Understanding the Interaction of Electromagnetic Waves with Metamaterials

Educational Research in Spain: A review through the Education Awards of CESEI - IEEE

Educational Software Interface for Power Electronic Applications

Educational visualizations of syntax error recovery 1019

Educative use of simulators in free software for the education of the physics $\quad 621$

in the engineering programs

Efectiveness of a Peer Mentoring Program in Engineering Education

Embedding Sustainability in Capstone Engineering Design Projects

Encouraging Interaction and Status Awareness in Undergraduate Software

Engineering Projects

Engaging Weaker Programmers in Problem Solving

Engineering Education in the Developing World: Complexity and Sustainability

Engineering in Latin America: A view at the Higher Education Level

Engineering societies as a vehicle tool for engineering students.

Engineers and their practice: a case study

Enhance Learning System by using Real-time Internet Classroom and Web-

Enhancing Authoring, Modelling and Collaboration in E-learning environments: UNED research outline in the context of E-Madrid excellence network

Enhancing Database Querying Skills by Choosing a More Appropriate

Interface 
ESA Hands-on Space Education Project Activities for University Students:

Attracting and Training the Next Generation of Space Engineers

Evolutionary algorithms for subgroup discovery applied to e-learning data

Evolutive Mechanism for E-Learning Platforms - A new approach for old

methods

Experiences in using a MUVE for enhancing motivation in engineering

education

Experiences in Using Integrated Multimedia Streaming Services to Support E-

Learning in Manufacturing Processes

Experiments in evaluation: towards an eXtreme Learning method

761

Federated authentication and authorization for reusable learning objects

1071

Filling the gap of Information Security Management inside ITIL ${ }^{\circledR}$ : proposals

for posgraduate students

Fingerprint Identification in LMS and its Empirical Analysis of Engineer

Students' Views

FPGA/Embedded system Training Kit Targeted to graduate Courses towards Industry level short training

Game-based learning in technology management education

GE3D: a virtual campus for a technology-enhanced learning

Generalization of an Active Electronic Notebook for Teaching Multiple

Programming Languages

GILABVIR: Virtual Laboratories and Remote Laboratories in Engineering.

Hands-on intelligent mobile robot laboratory with support from the industry

Hardware Implementation of Remote Laboratory for Digital Electronics

Higher Education Process Management Model, Educative Programs

Improvement in Software Quality

How can Apache help to teach and learn automatic control?

Illustrating amazing effects of optics with the computer

Impact of Learning Experiencies Using LEGO Mindstorms ${ }^{\circledR}$ in Engineering

Courses

Implantation of a Methodology based on Standard Supplements applied in

Engineering Education

Implementation of a virtual communications laboratory for e-Learning

Implementation of An Engineering Educator Graduation Program for the

formation of New Skilled Engineering Teachers

Implementing new learning methodologies in the Hard Sciences: a cross

curricular study of students' and professors acceptance

Indexing and Searching Learning Objects in a Peer-to-Peer Network

Influence of Libre Software in Education The blogs planet case

Influence of PBL Practical Classes in Microcontroller-Based Digital Systems

Learning

Information Technology in Logistics: Teaching Experiences, Infrastructure 
Innovative Learning and Teaching Methodology in Electronic Technology

Area. A Case Study in Computer Science University Degrees

Innovative Practices for Learning Human-Computer Interaction by

Engineering Learners

Integrating digital video resources in teaching e-learning engineering courses

Integrating People and Technology By Design: Design-First Instruction for

Introductory Students in Information Technology

Integrating Teams In Multidisciplinary Project Based Learning in Mechanical

Engineering

Integrating the Design Thinking into the UCD's methodology

Integration View of Web Labs and Learning Management Systems

1409

Intelligent evaluation in educational context

401

Internet-based Performance-centered Learning Environment for Curriculum

Support (IPLECS) and its application in mLearning

Interoperable Content for Performance in a Competency-driven Society:

Results from the iCoper project

Introducing alternative assessment into engineering language education at the

Madrid Technical University

Introducing multidisciplinary thinking in Computer Engineering - A new way

of teaching database systems

Introducing Project Management Theory into a Capstone Design Sequence

Introducing Scenario Based Learning: Experiences from an undergraduate

electronic and electrical engineering course.

Introduction to Electronics as a Minor Subject

Knowledge Management and organizational learning University-Company -

Learn to Learn-

Knowledge Management and Professional Profiles in Electronic Systems

Engineering

Knowledge, skills, and competences -- Descriptors for Engineering Education

Lab2go - A Repository to Locate Educational Online Laboratories

Learning by doing in Project Management: Acquiring skills through a

collaborative mode

Learning Dynamics and Control in a Virtual World

Learning engineering by teaching engineering in the European Higher

Education Area

Learning network protocols through WSN based games

LMS and Web 2.0 Tools for e-Learning: University of Deusto's

Experience.Taking Advantage of Both

M2Learn: Towards a homogeneous vision of advanced mobile learning development

Madar learning : learning envirnment for E\&M learning

Management and Optimal Distribution of Large Student Numbers 
Meaningful learning checking of concepts related to equations and functions

in Physics Chemistry according to the main theme gas laws.

Measuring collaboration and creativity skills through rubrics

Mechatronics E-course for regular students and adults: realization and comparison of efficiency

Meta-analysis of the TAEE project applying social network analysis

Methods of the quality assurance applied at remote laboratory selection

Mixed e-Assessment: an application of the student-generated questions technique

M-learning tools on distance education

Motivating Younger Students by Using Engineering Graduation Projects to

Facilitate their Work

Nano-World A Showcase Suite for Technology-Enhanced Learning

Natural Sciences in the Information Society - First Experiences

NETLAB: Online Laboratory Management System

New Directions in Engineering Accreditation

OCW Consortium: learning through the worldwide sharing and use of free, open, high-quality education materials organized as courses

OER's production cycle with social authorship and semantic tools

On Education Quality Control Issues for Sino-France Hybrid Engineer

Online assessment of practical knowledge in electronics laboratory

Open educational resources (OER) inspire teachers and motivate students

Personal Learning Environments in a Global Higher Engineering Education

Web 2.0 realm

Personalized Construction of Self-Evaluation Tests

Platform for teaching of location technologies based on Zigbee Wireless

Sensor Networks by learning-through-play theory

Portugol IDE v3.x

Practical Framework of Employability Skills for Engineering Graduate in Malaysia

Practice and Research in Engineering Education: Activities of the CESEI

Technical Committee

Principles for the Design of a Remote Laboratory 
Retaining electronic engineering students by project- and team-work from the

Reviews and Findings on Implementing Active Learning in a Large Class

Environment

Role of Faculty in Promoting Lifelong Learning: Characterizing Classroom

Environments

Role of regional consortia in OCWC: OCW-Universia

Scientific project management course introduction in doctoral studies in Riga Technical University

SecondLab: A Remote Laboratory under Second Life

Simulations in Undergraduate Electrodynamics

SOA-based Architecture for a Generic and Flexible E-assessment System

Some Research Questions and Results of UC3M in the E-Madrid Excellence

State-of-the-art simulation systems for information security education, training and awareness

Student Internship Placements. Improving the quality of engineering internship programs.

Student Motivation and Cross-curricula Development through e-learning applied to cooperation

Supporting Person-Centered Learning: Does the Choice of the Learning Management System Matter?

Supporting the Delivery of Learning Contents with Laboratory Activities in SAKAI

Teaching Digital and Analog Modulation to Undergradute Information Technology Students Using Matlab and Simulink

Teaching Microprocessors Design Using FPGAs 
Technological Development, Sustainability: Discussions about International

Aspects of Engineering Education

Telefónica University Chairs Network

The developing of personal and professional skills in automotive engineers

through university competitions

The development of professional mentoring for engineers undertaking a

workbased learning Masters degree

The influence of design problem complexity on the attainment of design skills and student perceptions

The LULA Project by the Telefónica Chair of the University of Extremadura - LULA Linux Distribution for Latin American Universities

The Montegancedo Astronomical Observatory. The first free remote observatory for learning astronomy

The New Degree in Materials Engineering at the Technical University of Madrid (UPM)

The Role of Superior Education Institutions on Post-Secondary (Non Superior) Education

The use of agents to represent learners in role-play training

The Use of Role Playing in Engineering Curricula: a Case Study in HumanAutomation Systems

Three Online Neutron Beam Experiments Based on the iLab Shared Architecture

TICTAC: Information and Communication Technologies for Augmentative Communication Boards

Tokyo Tech Graduate Program Allied with Thailand: TAIST (Thailand Advance Institute of Science and Technology) - Tokyo Tech

Tools for Collaborative Development of Visual Models and Languages

Towards the loose coupling between LMS and Remote Laboratories in Online Engineering Education

Toy Design Experience: Improving Student's Motivation and Results in a Final Year Subject

Training Microsystems Technologies in an European eLearning Environment

Trends of Use of Technology in Engineering Education

Use of Advanced Technologies in a RF and Microwave Engineering Course

Use of E-Learning functionalities: results of a survey along Spain

VenDASys - a versatile experimentation platform

Virtual analog and digital communications laboratory: LAVICAD

Virtual flute: electronic device that uses virtual reality to teach how to play a 
Web-based Time Schedule System for Multiple LMSs on the SSO/Portal Environment

Who enrolls in electrical engineering? A quantitative analysis of U.S.A. student trajectories

WikiDIS: a case of collaborative content management system for educative community

Wireless $4 x 4$ : an integrating learning experience for Telecommunications students

Workshop on VISIR electrical and electronic remote lab: Practical view

Workshop on VISIR electrical and electronic remote lab: Principles and

1663 educational view

yPBL methodology: a problem-based learning method applied to Software Engineering 